\title{
Chemical analysis of carbon stars in the Local Group
}

\section{The Small Magellanic Cloud and the Sagittarius dwarf spheroidal galaxy ${ }^{\star}$}

\author{
P. de Laverny ${ }^{1}$, C. Abia ${ }^{2}$, I. Domínguez ${ }^{2}$, B. Plez ${ }^{3}$, O. Straniero ${ }^{4}$, R. Wahlin ${ }^{5}$, K. Eriksson ${ }^{5}$, and U. G. Jørgensen ${ }^{6}$ \\ 1 Observatoire de la Côte d'Azur, Dpt. Cassiopée UMR6 202, 06304 Nice Cedex 4, France \\ e-mail: laverny@obs-nice.fr \\ 2 Dpto. Física Teórica y del Cosmos, Universidad de Granada, 18071 Granada, Spain \\ 3 GRAAL, UMR5024, Université de Montpellier II, 34095 Montpellier Cedex 5, France \\ 4 INAF - Osservatorio di Collurania, 64100 Teramo, Italy \\ 5 Department of Astronomy and Space Physics, Box 515, 75120 Uppsala, Sweden \\ ${ }^{6}$ Niels Bohr Institute, Astronomical Observatory, Juliane Maries vej 30, 2100 Copenhagen, Denmark
}

Received 12 May 2005 / Accepted 12 September 2005

\section{ABSTRACT}

We present the first results of our ongoing chemical study of carbon stars in the Local Group of galaxies. We used spectra obtained with UVES at the $8.2 \mathrm{~m}$ Kueyen-VLT telescope and a new grid of spherical model atmospheres for cool carbon-rich stars which include polyatomic opacities, to perform a full chemical analysis of one carbon star, BMB-B 30, in the Small Magellanic Cloud (SMC) and two, IGI95-C1 and IGI95-C3, in the Sagittarius Dwarf Spheroidal (Sgr dSph) galaxy. Our main goal is to test the dependence on the stellar metallicity of the s-process nucleosynthesis and mixing mechanism occurring in AGB stars. For these three stars, we find important s-element enhancements with respect to the mean metallicity $([\mathrm{M} / \mathrm{H}])$, namely $[\mathrm{s} / \mathrm{M}] \approx+1.0$, similar to the figure found in galactic AGB stars of similar metallicity. The abundance ratios derived between elements belonging to the first and second s-process abundance peaks, corresponding to nuclei with a magic number of neutrons $N=50(88 \mathrm{Sr}, 89 \mathrm{Y}, 90 \mathrm{Zr})$ and $N=82(138 \mathrm{Ba}, 139 \mathrm{La}, 140 \mathrm{Ce}, 141 \mathrm{Pr})$, agree remarkably well with the theoretical predictions of low mass $\left(M<3 M_{\odot}\right)$ metal-poor AGB nucleosynthesis models where the main source of neutrons is the ${ }^{13} \mathrm{C}(\alpha, n){ }^{16} \mathrm{O}$ reaction activated during the long interpulse phase, in a small pocket located within the He-rich intershell. The derived $\mathrm{C} / \mathrm{O}$ and ${ }^{12} \mathrm{C} /{ }^{13} \mathrm{C}$ ratios are, however, more difficult to reconcile with theoretical expectations. Possible explanations, like the extrinsic origin of the composition of these carbon stars or the operation of a non-standard mixing process during the AGB phase (such as the cool bottom process), are discussed on the basis of the collected observational constraints.

Key words. stars: abundances - stars: carbon - nuclear reactions, nucleosynthesis, abundances - galaxies: Local Group

\section{Introduction}

Asymptotic giant branch (AGB) stars are believed to be the main producers of s-elements in the Universe. Indeed, the s-process is activated during the late AGB phase, within the intershell region, when the He-burning shell suffers recurrent thermal instabilities (thermal pulses or TPs). After each TP, the convective envelope can penetrate inward in mass, dredging up the material enriched with the ashes of He-burning (third dredge up or TDU), mainly carbon and s-elements. Later on, this material is ejected through stellar winds, modifying the chemical composition of the interstellar medium (cf. Iben \& Renzini 1983; Busso et al. 1999).

* Based on observations collected with the VLT/UT2 Kueyen telescope (Paranal Observatory, ESO, Chile) using the UVES instrument (program ID 71.D-0107).
In intermediate mass AGB stars $\left(M \geq 3 M_{\odot}\right.$, IMS), free neutrons can be released at the base of the convective zone generated by a thermal pulse through the ${ }^{22} \mathrm{Ne}(\alpha, n)^{25} \mathrm{Mg}$ reaction (see e.g. Iben \& Renzini 1983). Indeed, in the He-rich intershell of these massive AGB, the ${ }^{22} \mathrm{Ne}$ is exposed to a temperature as high as $3.5 \times 10^{8} \mathrm{~K}$, which corresponds to an equilibrium density of about $10^{11}$ neutrons per $\mathrm{cm}^{-3}$. However, due to such a high neutron density, the resulting nucleosynthesis is characterized by the activation of several branchings along the s-process path, leading to an elemental distribution of the heavy nuclei that is rather different from those observed in the majority of the galactic MS, S and C (N type) stars (see e.g. Lambert et al. 1995; and Abia et al. 2000) and to an isotopic composition that is in conflict with those found in meteoritic SiC grains, which are presolar condensates formed in the outflows of carbon-rich AGB stars (e.g. Zinner 1998). On the contrary, 
in low mass AGB stars $\left(M<3 M_{\odot}\right.$, LMS $)$, the temperature within the He-rich intershell barely attains $3 \times 10^{8} \mathrm{~K}$ and the ${ }^{22} \mathrm{Ne}(\alpha, n)^{25} \mathrm{Mg}$ is only marginally activated. Nowadays, it is well accepted that the ${ }^{13} \mathrm{C}(\alpha, n){ }^{16} \mathrm{O}$ reaction is the main neutron source acting in LMS. It only requires $\sim 10^{8} \mathrm{~K}$ to be activated, a temperature usually attained at the top layer of the He-rich intershell during the interpulse periods. The extant theoretical models (Straniero et al. 1995; Straniero et al. 1997; Herwig et al. 1997; Gallino et al. 1998; Goriely \& Siess 2001) assume that a small amount of hydrogen is injected from the convective envelope into the intershell region during the TDU. At hydrogen reignition, owing to proton captures on ${ }^{12} \mathrm{C}, \mathrm{a}{ }^{13} \mathrm{C}$ pocket forms within the intershell. Then, the ${ }^{13} \mathrm{C}$ is fully consumed by $\alpha$ captures during the interpulse phase, and then leads to a substantial s-process nucleosynthesis with a peak neutron density never exceeding $10^{7} \mathrm{~cm}^{-3}$. The freshly synthesized s-elements are finally engulfed by the convective shell generated by the next TP. The marginal activation of the ${ }^{22} \mathrm{Ne}$ neutron source may slightly modify the s-element distribution within the He-rich intershell. The enhancement of the s-elements revealed by spectroscopic studies of MS, S, SC and C-stars confirms the occurrence of repeated TDU episodes.

One of the most important theoretical results concerning this new s-process nucleosynthesis paradigm is its critical dependence on the stellar metallicity and mass. Current models (e.g. Busso et al. 1999; Goriely \& Siess 2001) show that the predicted relative abundances of the s-peak nuclei $(\mathrm{Zr}, \mathrm{Ba}$ and $\mathrm{Pb}$ ) vary according to the stellar metallicity. At low metallicity, the flow along the s-path drains the $\mathrm{Zr}$ and Ba peaks and builds an excess at the doubly magic ${ }^{208} \mathrm{~Pb}$, which is at the termination of the s-path. Thus, as the metallicity of the AGB star decreases, models predict larger $\mathrm{Pb} / \mathrm{Ba} / \mathrm{Zr}$ ratios. However, for a given metallicity, a spread in the $\mathrm{Pb} / \mathrm{Ba} / \mathrm{Zr}$ ratios indicates a spread in the amount of ${ }^{13} \mathrm{C}$, which drives the neutron production and the subsequent s-process nucleosynthesis in a LMS (Gallino et al. 1998; Delaude et al. 2004). The s-elements abundance pattern found in the metal-poor Pb-rich stars (Van Eck et al. 2001, 2003; Aoki et al. 2002) and, at higher metallicities, in post-AGB stars (Reyniers et al. 2004) points to the existence of such a spread.

A comfortable agreement between nucleosynthesis calculations and s-element abundance patterns derived in AGB stars of different types in the solar neighborhood has been found (e.g. Busso et al. 2001). However, such comparisons mainly concern extrinsic stars (i.e. stars that owe their chemical peculiarities probably to mass transfer in a binary system) belonging to the disk of our Galaxy. Only in a few cases has the predicted chemical pattern in intrinsic AGB or post-AGB stars (i.e. stars that owe their chemical peculiarities to an in situ nucleosynthesis and mixing processes) in a range of metallicity been checked (Reyniers et al. 2004; Domínguez et al. 2004; Abia et al. 2002). Note that metal-poor AGB belonging to the old galactic halo have such small masses $\left(0.6 M_{\odot}\right.$ on the average) that the TDU cannot take place (Straniero et al. 2003).

Due to their different chemical evolution histories, the satellite galaxies have stellar populations with metallicities covering a wide range $(-3.0 \leq[\mathrm{Fe} / \mathrm{H}] \leq$ 0.0, see e.g. Groenewegen 2004; Shetrone et al. 1998;
Bonifacio et al. 2004a). Therefore, the study of AGB stars in the Local Group of galaxies provides an alternative sample of intrinsic metal-poor and relatively young AGB stars. Most importantly, since the distance of these external stellar systems is well known, a more accurate determination of the stellar luminosity can be estimated. Furthermore, there is observational evidence that in many of these galaxies the star formation histories extended at least until a few Gyr ago (e.g. Demers et al. 2003; Battinelli et al. 2003; Arimoto et al. 2004; Domínguez et al. 2004; Rizzi et al. 2004). This increases the probability of observing in these stellar systems metal-poor thermally pulsing AGB (TP-AGB) stars, whose abundance pattern is being modified by the occurrence of the TDUs (intrinsic AGBs). Last but not least, the knowledge of the contribution of these low metallicity AGB stars to the chemical evolution of their parent galaxies provides new hints to discriminate between alternative scenarios of galactic formation (Venn et al. 2004): were the satellite galaxies the basic building blocks of our own Galaxy? (e.g. Bullock et al. 2001), or are they debris of larger systems whose structure and evolution have been altered by their proximity to our Galaxy (e.g. Grebel et al. 2003)?

In this paper we report the first chemical analysis of extragalactic low-metalicity carbon stars found in the Small Magellanic Cloud and the Sagittarius dwarf spheroidal galaxy. The selected stars are presented in Sect. 2 together with the observations. We describe in Sect. 3 the chemical analysis performed. Then, by analyzing the derived abundances and in particular those of the s-elements versus metallicity, we provide constraints to the evolutionary status of the studied stars and discuss our results in the framework of the current AGB nucleosynthesis models at low metallicity.

\section{Star selection and observations}

From the available catalogs of carbon-rich stars in external galaxies (e.g. Groenewegen 2002), we first selected the brightest candidates that are spectroscopically confirmed as carbonrich and as members of their host galaxy. Host galaxies have been selected to span a large range of metallicities, in order to provide better constraints to the nucleosynthesis models of AGB stars. In this first study we present the spectroscopic results for three carbon stars, one belonging to the Small Magellanic Cloud (SMC) and two stars to the Sagittarius dwarf spheroidal galaxy (Sgr dSph). Table 1 shows the photometric properties of our programme stars.

The SMC is an irregular galaxy at a distance of about $63 \mathrm{kpc}$ (Cioni et al. 2000) and suffering an interstellar extinction $A_{\mathrm{B}}=0.16^{\mathrm{mag}}$ (NASA/IPAC database, Schlegel et al. 1998). Its mean metallicity is around $[\mathrm{M} / \mathrm{H}]=-0.74^{1}$ (Luck et al. 1998) although lower metallicities (e.g. -1.05) are also reported (Rolleston et al. 1999). The selected star, BMB-B 30 (B, standing for the bar of the SMC), was first identified as carbon-rich by Blanco et al. (1980). Its carbon-rich nature was

\footnotetext{
${ }^{1}$ Here we use the standard notation for the chemical abundance ratio of any element $X,[X / \mathrm{H}]=\log (X / \mathrm{H})_{\star}-\log (X / \mathrm{H})_{\odot}$ where $\log (\mathrm{H}) \equiv 12$ is the abundance of hydrogen by number. In this scale the abundance of any element $X$ is noted as $\epsilon(X)$.
} 
Table 1. Carbon stars observations log and properties.

\begin{tabular}{cccccccc}
\hline \hline Star & Date obs. & Exp. time $(\min )$ & $B_{J}$ & $R$ & $J$ & $H$ & $K$ \\
\hline Sag IGI95-C1 & $2003-07-03$ & 195 & $16.9^{a}$ & $14.6^{a}$ & $12.3^{b}$ & $11.4^{b}$ & $11.1^{b}$ \\
Sag IGI95-C3 & 2003-07-03/04/06 & 195 & $17.3^{a}$ & $14.3^{a}$ & $11.3^{b}$ & $10.0^{b}$ & $9.4^{b}$ \\
SMC BMB-B 30 & 2003-06-11 and 2003-07-05/30/31 & 195 & - & $14.3^{c}$ & $12.1^{d}$ & $11.1^{d}$ & $10.7^{d}$ \\
\hline
\end{tabular}

References: ${ }^{a}$ Ibata et al. (1995); ${ }^{b}$ Whitelock et al. (1996); ${ }^{c}$ Blanco et al. (1980); ${ }^{d}$ Two Micron All Sky Survey.

later confirmed by Rebeirot et al. (1993) and, since the survey of Smith et al. (1995), it was also known to be non-enhanced in lithium.

The Sgr dSph galaxy was discovered ten years ago by Ibata et al. (1995). It lies at about $26 \mathrm{kpc}$ from the Sun (Monaco et al. 2004) and is characterized by a large spread in metallicity, $-0.8 \leq[\mathrm{Fe} / \mathrm{H}] \leq 0.0$ (see e.g. Bonifacio et al. 2004b), and recent observations reveal the existence of stars with metallicity as low as $[\mathrm{Fe} / \mathrm{H}] \approx-3$ (Bonifacio et al. 2004a). The adopted interstellar extinction is $A_{\mathrm{B}}=0.66^{\mathrm{mag}}$ (NASA/IPAC database, Schlegel et al. 1998). The two selected stars were confirmed to be carbon-rich and members of the Sgr dSph galaxy by Ibata et al. (1995). According to Whitelock et al. (1996), IGI95-C3, is one of the brightest star in Sgr dSph, with extremely red colors suggesting a large mass-loss rate and/or a low effective temperature (see below the problems encountered during its chemical analysis).

To date, the spectral type of the three carbon stars is unknown since their full spectrum has never been observed. We therefore cannot use common indicators as the relative intensity of molecular bands of $\mathrm{C}_{2}$ and $\mathrm{CN}$ to classify them (such indicators lie outside our observed spectral ranges). We have estimated their bolometric luminosity using the calibrations by Alksnis et al. (1998) from $M_{K}$ (see Table 3). These calibrations are obtained from studies of galactic carbon stars with known distances from Hipparcos parallax measurements. It is not clear whether these calibrations can be safely applied to metal-poor carbon $(\mathrm{C})$ stars such as those studied here. Nevertheless, using the bolometric corrections of $M_{K}$ from the $(J-K)_{0}$ index for carbon stars in the SMC by Wood et al. (1983), we obtain very similar luminosities (see Table 3). The bolometric magnitudes obtained for BMB-B 30 and IGI95-C3 are compatible with the hypothesis that they are TP-AGB stars undergoing TDU. IGI95-C1 seems too faint to be an intrinsic C-star, even considering that the minimum luminosity at which an AGB star becomes carbon-rich is lower at low metallicity (Straniero et al. 2003). In fact, by using a simple core mass-luminosity relation to constraint the initial luminosity of a thermally pulsing AGB phase (Paczynski 1970), and adopting $M_{\mathrm{H}}=0.55 M_{\odot}$ for the mass of the $\mathrm{H}$-exhausted core, one gets a minimum bolometric magnitude of about -4 . In any case, the bolometric magnitudes shown in Table 3 are affected by large uncertainties. As pointed out by Guandalini et al. (2006), with an effective temperature of $\sim 3000 \mathrm{~K}$, most of the energy radiated by a C-star is in the mid infrared wavelength range. By using IR data, these authors conclude that the galactic carbon stars are, in many cases, 1 or 2 bolometric magnitudes brighter than reported in the available catalogs (Groenewegen 2002).
The three selected targets were observed in service mode with the UVES spectrograph attached to the second VLT unit (Kueyen telescope) in June and July 2003. The spectra have been collected using the UVES standard settings Dichroic 2,CD\#2 centered at $4370 \AA$ and $C D \# 4$ centered at $8600 \AA$, leading to observed spectral domains from $\sim 4200$ to $\sim 5000 \AA$ and from $\sim 6700$ to $\sim 9000 \AA$. The slit width was $1^{\prime \prime}$, corresponding to a resolving power of about 40000 . The UVES Data Reduction Standard Pipeline was used for the reduction of the spectra. Then, for each star and spectral range, the spectra were first averaged and then binned by three pixels as well as corrected to the local standard of rest. Finally, all the spectra were normalized to a local continuum by fitting a polynomial connecting the higher flux points in the spectral regions studied. For this procedure and, as a guide, we used the continuum location that may be inferred looking at the spectral atlas of carbon stars of different types by Barnbaum et al. (1996) and, in particular, in the $8000 \AA$ region the theoretically expected continuum windows identified by Wyller (1966) were used as reference points. Due to the huge number of atomic and molecular lines used for the synthetic spectra calculation, it is reasonable to think that the theoretical continuum points are not too far from the true continuum. Indeed, our theoretical spectra show maximum flux points at these wavelengths. It was never necessary to modify the initial placement of the continuum by more than $\pm 5 \%$. Errors introduced by this uncertainty in the continuum position were taken into account. However, systematic errors due to a larger uncertainty in the continuum location cannot be completely discarded (see below).

\section{Analysis: the extragalactic carbon stars and their chemical properties}

We have used the method of spectral synthesis in LTE to derive the chemical abundances of the sample stars with a special emphasis on specific spectral regions. In particular, the selected regions were: (i) between 4750-4950 $\AA$ mainly for s-elements and the mean metallicity ${ }^{2}$; (ii) 6700-6730 for $\mathrm{Li}$; (iii) 7050-7080 $\AA$ for some $\mathrm{Ti}$ lines and one Sr line; (iv) 7780-7820 $\AA$ for $\mathrm{Rb}$ and two Ni lines and 7990-8040 ̊ to derive the carbon isotopic ratio. The adopted atomic line list is basically that used in Abia et al. (2001, 2002). We have added some lines taken from the atomic data bases of Kurucz (CD-ROM No. 13) and VALD (Kupka et al. 1999). Some revisions have been made using solar gf-values derived by Thévenin (1989, 1990), and the

\footnotetext{
2 The mean metallicity of the stars $[\mathrm{M} / \mathrm{H}]$ (Table 3 ) was obtained as the mean value derived from several lines of Fe, Ti, V, Ni, $\mathrm{Cr}$ and $\mathrm{Mn}$.
} 
Table 2. Spectroscopic parameters of the new identified lines.

\begin{tabular}{cccr}
\hline \hline Ion & $\lambda(\AA)$ & $\chi(\mathrm{eV})$ & \multicolumn{1}{c}{$\log g f$} \\
\hline Zn I & 4810.52 & 4.08 & -0.170 \\
Nb I & 4802.44 & 0.08 & -1.900 \\
Ru I & 4757.84 & 0.93 & -0.280 \\
Ru I & 4769.30 & 0.81 & -1.740 \\
Ru I & 4844.56 & 1.12 & -0.810 \\
Pr II & 4801.13 & 0.48 & -0.878 \\
Pr II & 4837.03 & 0.20 & -1.464 \\
Pr II & 4848.52 & 0.05 & -1.584 \\
Gd I & 4758.70 & 0.88 & 0.045 \\
Hf I & 4800.50 & 0.70 & -1.210 \\
W I & 4843.84 & 0.41 & -0.990 \\
\hline
\end{tabular}

theoretical estimates by $\mathrm{Xu}$ et al. (2003) and Den Hartog et al. (2003) for the Nd II lines, and the DREAM data base (http://w3.umh.ac.be/ astro/dream.shtml) for $\mathrm{Sm}$ and Ce singly ionized lines. With respect to Abia et al. (2001, 2002), we have identified additional spectral features corresponding to elements whose abundances are derived here (see Table 2).

The molecular line list includes $\mathrm{CN}, \mathrm{C}_{2}, \mathrm{CH}$ and $\mathrm{MgH}$ lines with the corresponding isotopic variations. $\mathrm{C}_{2}$ lines are from Querci et al. (1971). CN and CH lists were assembled from the best available data and are described in Hill et al. (2002) and Cayrel et al. (2004). MgH lines come from Kurucz (CD-Rom No. 13). In particular, the $\mathrm{MgH}$ lines, which were not included in Abia et al. (2001, 2002), act like a pseudo-continuum below $4800 \AA$. This might be significant if the star under analysis is found to be $\alpha$-enhanced ( $[\mathrm{Mg} / \mathrm{Fe}]>0$ ), as one would expect for metal-poor stars according to the trend found in our Galaxy. However, as far as we know, all the chemical analysis so far performed in the SMC and the Sgr dSph stellar populations do not reveal a clear $\alpha$-enhancement trend. Thus, we have adopted in the analysis no enhancement, i.e. $[\mathrm{Mg} / \mathrm{Fe}]=0.0$. The ratio $[\mathrm{Ca} / \mathrm{M}]$ derived in two stars (see Table 4 ) is compatible with this figure although we note that our $\mathrm{Ca}$ abundance estimation is made only from one line, CaI $6717 \AA$. We checked our line list by comparing theoretical with observed spectra of the Sun and Arcturus. The comparison with Arcturus allowed us to check some molecular lines whereas most of these features are not visible in the Solar spectrum. For the Sun, we used the semi-empirical model atmosphere by Holweger \& Müller (1974) with abundances from Grevesse \& Sauval (1998). For Arcturus we computed a model atmosphere with main parameters according to Decin et al. (2000) and abundances from Peterson et al. (1993). Theoretical spectra were computed with the turbospectrum code (Alvarez \& Plez 1998, and further improvements by Plez) in spherical geometry. The comparisons showed an excellent agreement with the solar spectrum in all the spectral ranges studied, although the comparison was not as good in the case of Arcturus in the 4750-4950 $\AA$ region. This indicates that our atomic and molecular line list is still not complete in this spectral range (despite the fact that we included about 100000 lines in this region) and/or the line data of some molecules are partially erroneous. Most of the spectral features used in the chemical analysis of our stars, nevertheless, are well fitted in the Arcturus spectrum.

The effective temperatures were first estimated from the calibrations of the $(V-K)_{0}$ and $(J-K)_{0}$ color indexes by Aaronson \& Mould (1985). However, the estimated values are just used as a starting point, the final values adopted in Table 3 being obtained through an iterative process by comparing observed and theoretical spectra computed with different effective temperatures. It is well known that AGB stars are variable and, hence, their $T_{\text {eff }}$ may change during the pulsation. The photometric calibration used to estimate the effective temperature indicates that the uncertainty in our $T_{\text {eff }}$ is certainly not lower than $\pm 250 \mathrm{~K}$. We also note that effective temperature estimates from $(V-K)$ may be affected by stellar variability since the measurements in the two bands were obtained on different occasions. Nevertheless, the final adopted values agree quite well with those derived from the photometric calibrations within $\pm 100 \mathrm{~K}$. We set the gravity at $\log g=0.0$ for all the stars, following Lambert et al. (1986) who to a considerable degree based their choice on the luminosities and masses of carbon stars in the Magellanic Clouds. For the microturbulence, we adopted $\xi=2.2 \mathrm{~km} \mathrm{~s}^{-1}$, which is a value suitable for AGB stars (Lambert et al. 1986). Theoretical spectra were convolved using Gaussian functions with a $F W H M$ value according to the instrumental profile $(\sim 0.15 \AA)$ and macroturbulence velocities ranging from 4 to $7 \mathrm{~km} \mathrm{~s}^{-1}$. Uncertainties in the abundances derived due to changes in gravity, microturbulence and macroturbulence within $\pm 0.5 \mathrm{dex}, \pm 0.20 \mathrm{~km} \mathrm{~s}^{-1}$ and $\pm 1 \mathrm{~km} \mathrm{~s}^{-1}$, respectively, were considered.

Apart from $T_{\text {eff }}$, the main atmospheric parameter affecting theoretical spectra of carbon-rich stars is the $\mathrm{C} / \mathrm{O}$ ratio. We estimated this ratio through an iterative process, by comparing observed and theoretical spectra computed for different values of the carbon abundance, while keeping the other atmospheric parameters constant. For that purpose, we mainly considered the region near $8000 \AA$ since it is the most sensitive to changes in the $\mathrm{C} / \mathrm{O}$ ratio. Indeed, the spectrum of $\mathrm{C}$-stars in this region is mostly affected by $\mathrm{CN}$ and much weaker $\mathrm{C}_{2}$ absorptions. We could not derive the carbon abundance directly from $\mathrm{C}_{2}$ lines since (i) our linelist is not enough accurate around the $8000 \AA$ region (see Plez \& Cohen 2005); (ii) the $C_{2}$ Swan $(0,0)$ band around $5160 \AA$ was not observed; and (iii) the collected spectra in the blue part become too noisy to consider the $\mathrm{C}_{2}$ Swan $(1,0)$ band around $4735 \AA$. We thus derive the carbon abundance from the $\mathrm{CN}$ lines assuming that $[\mathrm{N} / \mathrm{Fe}]=0.0$. We checked in previous works (Abia \& Isern 1996) that the $\mathrm{C} / \mathrm{O}$ ratio derived from the $8000 \AA$ range is almost insensitive to the assumed abundance of nitrogen within \pm 0.3 dex. However, the derived $\mathrm{C} / \mathrm{O}$ ratios have an additional uncertainty due to the adopted $\mathrm{O}$ abundance which we cannot determine independently. This is because theoretical spectra are almost insensitive to a large variation in the $\mathrm{O}$ abundance provided that the difference $\epsilon(\mathrm{C})-\epsilon(\mathrm{O})$ is kept constant. Indeed, when adding equal amounts of carbon and oxygen to the atmosphere, the sole effect in the outer layers is to increase the abundance of the $\mathrm{CO}$ molecule which has a negligible effect on the atmospheric structure. Therefore, this ambiguity allows a range of 
Table 3. Main characteristics of the extragalactic C-stars. We give the stellar name, the effective temperature, the mean metallicity $[\mathrm{M} / \mathrm{H}]$, the carbon over oxygen abundances ratio $(\mathrm{C} / \mathrm{O})$, the corresponding difference between carbon and oxygen abundances $(\epsilon(\mathrm{C})-\epsilon(\mathrm{O}))$, the carbon isotopic ratio and the range of estimated bolometric absolute magnitudes.

\begin{tabular}{ccccccc}
\hline \hline Star & $T_{\text {eff }}(\mathrm{K})$ & {$[\mathrm{M} / \mathrm{H}]$} & $\mathrm{C} / \mathrm{O}^{a}$ & $\epsilon(\mathrm{C})-\epsilon(\mathrm{O})$ & ${ }^{12} \mathrm{C} /{ }^{13} \mathrm{C}$ & $M_{\mathrm{bol}}{ }^{b}$ \\
\hline IGI95-C1 & 3300 & -0.80 & $1.18-1.06$ & 7.23 & 25 & $-3.1,-3.3$ \\
IGI95-C3 & 2900 & -0.50 & $1.10-1.05$ & 7.33 & 40 & $-4.5,-4.5$ \\
BMB-B 30 & 3000 & -1.00 & $1.20-1.09$ & 7.35 & $>300$ & $-5.6,-5.1$ \\
\hline
\end{tabular}

${ }^{a}$ The $\mathrm{C} / \mathrm{O}$ ratios indicate the range of values estimated from the analysis of the different spectral ranges (see text).

${ }^{b}$ The first value indicates the luminosity obtained from $M_{K}$ according to the calibration by Alknis et al. (1998). The second one was derived after the bolometric correction by Wood et al. (1983). See Sect. 2 for more details.

Table 4. Summary of the abundances derived in the programme stars. $N$, indicates the number of lines utilized for a specific element if more than one line was used (we then give the mean abundance together with its dispersion). [el/M] is the abundance ratio with respect to the mean derived $[\mathrm{M} / \mathrm{H}]$ (see text and Table 3 ) and is only quoted for elements with $A \geq 30$.

\begin{tabular}{|c|c|c|c|c|c|c|c|c|c|}
\hline & & IGI95-C1 & & & IGI95-C3 & & & BMB-B 30 & \\
\hline Element & $N$ & $\log \epsilon$ & {$[\mathrm{el} / \mathrm{M}]$} & $\bar{N}$ & $\log \epsilon$ & {$[\mathrm{el} / \mathrm{M}]$} & $N$ & $\log \epsilon$ & {$[\mathrm{el} / \mathrm{M}]$} \\
\hline$\overline{\mathrm{Li}}$ & & $<-0.50$ & & & -1.00 & & & -0.80 & \\
\hline $\mathrm{Ca}$ & & - & & & 5.85 & & & 5.30 & \\
\hline $\mathrm{Ti}$ & 3 & $4.20 \pm 0.12$ & & 2 & $4.70 \pm 0.05$ & & 4 & $3.90 \pm 0.08$ & \\
\hline V & 3 & $3.10 \pm 0.15$ & & 3 & $3.50 \pm 0.15$ & & & - & \\
\hline $\mathrm{Cr}$ & 2 & $4.90 \pm 0.10$ & & & 5.32 & & 2 & $4.98 \pm 0.05$ & \\
\hline $\mathrm{Mn}$ & & - & & & - & & 3 & $4.39 \pm 0.05$ & \\
\hline $\mathrm{Fe}$ & & 6.85 & & & _- & & 3 & $6.50 \pm 0.08$ & \\
\hline $\mathrm{Ni}$ & 2 & $5.50 \pm 0.10$ & & 2 & $5.85 \pm 0.05$ & & & 5.25 & \\
\hline $\mathrm{Zn}$ & & 4.00 & 0.20 & & 4.10 & 0.00 & & 4.00 & 0.40 \\
\hline $\mathrm{Rb}$ & & $<2.10$ & $<0.50$ & & - & & & 1.40 & 0.00 \\
\hline $\mathrm{Sr}$ & 2 & $3.00 \pm 0.17$ & 0.80 & 2 & $3.35 \pm 0.05$ & 0.90 & 2 & $2.55 \pm 0.15$ & 0.60 \\
\hline Y & 2 & $2.05 \pm 0.03$ & 0.60 & & 2.55 & 0.80 & 2 & $1.65 \pm 0.02$ & 0.40 \\
\hline $\mathrm{Zr}$ & 4 & $2.30 \pm 0.09$ & 0.50 & 5 & $3.30 \pm 0.20$ & 1.20 & 4 & $2.00 \pm 0.04$ & 0.40 \\
\hline $\mathrm{Nb}$ & & $<0.72$ & $<0.10$ & & $<1.20$ & $<0.30$ & & $<0.42$ & $<0.00$ \\
\hline $\mathrm{Ru}$ & 2 & $1.50 \pm 0.10$ & 0.50 & & - & & & 1.45 & $<0.60$ \\
\hline $\mathrm{Ba}$ & & 2.80 & 1.50 & & $2.55^{a}$ & 0.80 & & 2.60 & 1.50 \\
\hline $\mathrm{La}$ & 5 & $1.95 \pm 0.20$ & 1.60 & & 1.35 & 0.70 & 2 & $1.50 \pm 0.15$ & 1.40 \\
\hline $\mathrm{Ce}$ & & $<2.50$ & $<1.70$ & & - & & 2 & $1.60 \pm 0.20$ & 1.00 \\
\hline $\operatorname{Pr}$ & 4 & $1.10 \pm 0.04$ & 1.20 & & - & & 2 & $0.80 \pm 0.08$ & 1.10 \\
\hline $\mathrm{Nd}$ & 5 & $2.50 \pm 0.12$ & 1.80 & 2 & $2.00 \pm 0.05$ & 1.00 & 4 & $2.10 \pm 0.11$ & 1.60 \\
\hline $\mathrm{Sm}$ & 2 & $1.40 \pm 0.01$ & 1.20 & 2 & $1.50 \pm 0.10$ & 0.90 & 2 & $1.05 \pm 0.05$ & 0.95 \\
\hline $\mathrm{Gd}$ & & $<1.50$ & $<1.20$ & & - & & & - & \\
\hline Hf & & 1.00 & 0.90 & & - & & & 1.10 & 1.20 \\
\hline W & & 1.20 & 0.90 & & - & & & 0.50 & 0.40 \\
\hline
\end{tabular}

${ }^{a}$ The Ba abundance derived in this star may be affected by the presence of Merrill-Sanford bands (see text).

oxygen abundances and $\mathrm{C} / \mathrm{O}$ ratios giving almost identical synthetic spectra. Nevertheless, even considering an uncertainty of a factor three in the oxygen abundance, the $\mathrm{C} / \mathrm{O}$ ratios derived in our stars are found in the range $1<\mathrm{C} / \mathrm{O}<1.5$. On the other hand, and as already found by Abia et al. (2002), the derived $\mathrm{C} / \mathrm{O}$ ratio slightly decreases with the wavelength of the spectral region fitted. For instance, in BMB-B 30, we found a best value of $\mathrm{C} / \mathrm{O}=1.2$ at $\sim 8000 \AA$, but $\mathrm{C} / \mathrm{O}=1.09$ at $\sim 4800 \AA$. Similar differences are obtained for the other two program stars. The reason of this discrepancy is unknown. It may be related to the continuous opacity of the model atmosphere or to an incomplete or erroneous molecular line list. Note that a modification of the continuum location by $\sim 15 \%$ in the $4750-4950 \AA$ range would increase our derived $\mathrm{C} / \mathrm{O}$ ratio in this region by only a few hundredth of dex. Thus, the discrepancy would remain. The range in the $\mathrm{C} / \mathrm{O}$ ratios derived for each star is given in Table 3 as well as the corresponding abundance difference $\epsilon(\mathrm{C})-\epsilon(\mathrm{O})$.

Regarding the model atmospheres, we used a new grid of models for cool carbon-rich stars. The spherically symmetric model atmospheres were calculated with the MARCS code, using opacity sampling in 11000 frequency points. Atomic, diatomic and polyatomic $\left(\mathrm{C}_{2} \mathrm{H}_{2}, \mathrm{HCN}\right.$ and $\left.\mathrm{C}_{3}\right)$ absorptions were included. The microturbulence parameter was set to $2-3 \mathrm{~km} \mathrm{~s}^{-1}$. Turbulence pressures were neglected. Convection was included according to the local mixing-length recipe and found to be insignificant. The masses of the models were set to $2 M_{\odot}$, following Lambert et al. (1986). The abundance difference $(\epsilon(\mathrm{C})-\epsilon(\mathrm{O}))$ and the overall metallicity were varied. The adopted solar abundances for $\mathrm{C}, \mathrm{N}$ and $\mathrm{O}$ in the models are 


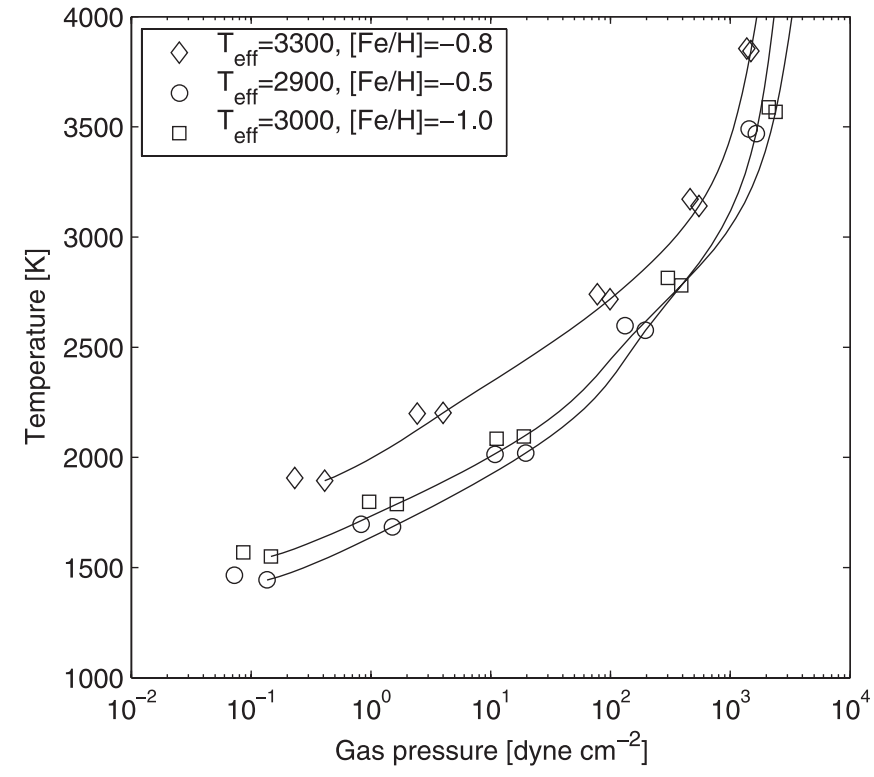

Fig. 1. Temperature structures of the model atmospheres computed with the stellar parameters corresponding to the three stars studied in this work. The points where the optical depth $\log \tau_{\text {Ross }}=-4,-3, \ldots, 0$ are indicated by markers. The models corresponding to IGI95-C1 are marked with diamonds, IGI95-C3 with circles and BMB-B 30 with squares. The structure is very sensitive to the carbon excess. The structures computed with the lower value of $\mathrm{C} / \mathrm{O}$ in Table 3 are plotted with solid lines and markers while the structures with high $\mathrm{C} / \mathrm{O}$ are indicated with markers only.

8.41, 7.80 and 8.67 according to the recent revision by Asplund et al. (2005). As mentioned previously, the models were found to be quite insensitive to $\epsilon(\mathrm{O})$ as long as $(\epsilon(\mathrm{C})-\epsilon(\mathrm{O}))$ was unchanged. The typical extension (from optical depths $10^{-4}$ to $10^{2}$ in $\tau_{\text {Ross }}$ ) was $7 \%$ of the total stellar radius. The temperature structures for the models used for the three stars are presented in Fig. 1. Further details will be given in forthcoming papers by Gustafsson et al. (2005) and Jørgensen et al. (2005). For each star we chose from the grid a specific model atmosphere with a given value of $T_{\text {eff }}, \mathrm{C} / \mathrm{O}$ and the metallicity and proceeded iteratively by changing these parameters until a good fit (by eye) in all the spectral regions was found. Then, the abundances of the other chemical species (s-elements, Li, etc.) were changed to fit specific spectral features.

A full discussion of the sources of error in the derived absolute abundances and element ratios due to uncertainties in the atmospheric parameters, continuum location $( \pm 5 \%)$ and random errors when a given element is represented by a few lines, as in our case, can be found in Abia \& Isern (1996) and Abia et al. $(2001,2002)$ and will not be repeated here. These errors range from \pm 0.2 dex for Ba to \pm 0.45 dex for Ce. We estimate a typical uncertainty in the mean metallicity, $[\mathrm{M} / \mathrm{H}]$, of \pm 0.30 dex. The typical error for the elemental ratios with respect to the metallicity $([X / \mathrm{M}])$ range between $0.30-0.40 \mathrm{dex}$, since some of the uncertainties cancel out when deriving this ratio. For the same reason, the error in the abundance ratio between elements $([X / Y])$ is somewhat lower, \pm 0.30 dex. For $\mathrm{Nb}$ and $\mathrm{Gd}$, the identified spectral features are very weak and blended, thus in some cases we can only establish upper limits.
We estimate an uncertainty in the carbon isotopic ratio of \pm 9 (see Abia \& Isern 1996). These numbers do not include possible systematic errors as N-LTE effects or an uncertainty larger than $5 \%$ in the continuum location (see below).

The abundances derived in IGI95-C3 from the 4850-4950 А region, merit a special note of caution. We found many difficulties to fit this spectral region. In fact, in this region several choices of $T_{\mathrm{eff}}, \mathrm{C} / \mathrm{O}$ ratio and metallicity can lead to a similar match of the observed spectrum. Finally we adopt the parameters shown in Table 3 as the best ones for IGI95-C3 (with $\mathrm{C} / \mathrm{O}=1.05$ ) but, in any case, we cannot obtain as good a fit as that obtained for BMB-B 30 and IGI95-C1 in this spectral region. We believe that this may be caused by the presence of Merrill-Sanford bands $\left(\mathrm{SiC}_{2}\right)$ in this star. These molecular bands are identified between 4100-5500 in carbon stars (e.g. Sarre et al. 2000; Yamashita \& Utsumi 1968; and McKellar 1947). In fact we detect extra-absorptions around 4867 and $4906 \AA$, not present in the other stars of the sample, which coincides with the position of some band heads of this molecule. Merrill-Sanford bands are indeed detected in this spectral region in some galactic C-stars with $T_{\text {eff }}<3100 \mathrm{~K}$ (Bergeat et al. 2001; Morgan et al. 2004), and our estimated effective temperature for this $\operatorname{star}\left(T_{\text {eff }}=2900 \mathrm{~K}\right.$, see Table 3 ) agrees with this figure. This means that the derived abundances in IGI95-C3 from the region 4850-4950 A have to be considered with caution. Obviously, Merrill-Sanford bands do not affect the abundances derived in this star from the other spectral ranges.

\section{Results and discussion}

Figures 2 and 3 show examples of theoretical fits to spectral regions from where most s-element abundances are derived. The fits are reasonably good despite the line lists used are likely incomplete. We are able to obtain a reasonable reproduction of some specific spectral features representative of single heavy elements. As noted above, for several elements the abundance is derived from just one line. Table 4 summarizes the abundances derived for individual elements in the programme stars. In the case that more than one line was used in the analysis we derived the mean value and its dispersion.

The three stars have low $\mathrm{Li}$ abundances, similar to that typically found in large $\mathrm{Li}$ abundance surveys of galactic C-stars (Denn et al. 1991; Abia et al. 1993). The Li content in BMB-B 30 was already studied by Smith et al. (1995) and their estimate agrees with the value given in Table 4. On the other hand, the mean metallicity derived in the stars, are compatible with the typical metallicity ranges derived from the stellar populations of these satellite galaxies in other observational studies (see references in Sect. 1). In particular, the metallicity derived for BMB-B 30, belonging to the SMC, confirms the low values previously reported by Rolleston et al. (1999) as the mean metallicity for this galaxy. On the basis of the derived $\mathrm{Ca}$ and $\mathrm{Ti}$ abundances, and considering the error bars, we do not find evidence of $\alpha$-enhancement in any of the stars studied.

It should be remarked that this is the first time that species like $\mathrm{Zn}, \mathrm{Ru}$, Hf and $\mathrm{W}$ are detected and measured in giant carbon stars. We find a moderate overabundance of $\mathrm{Zn}$ in all the 

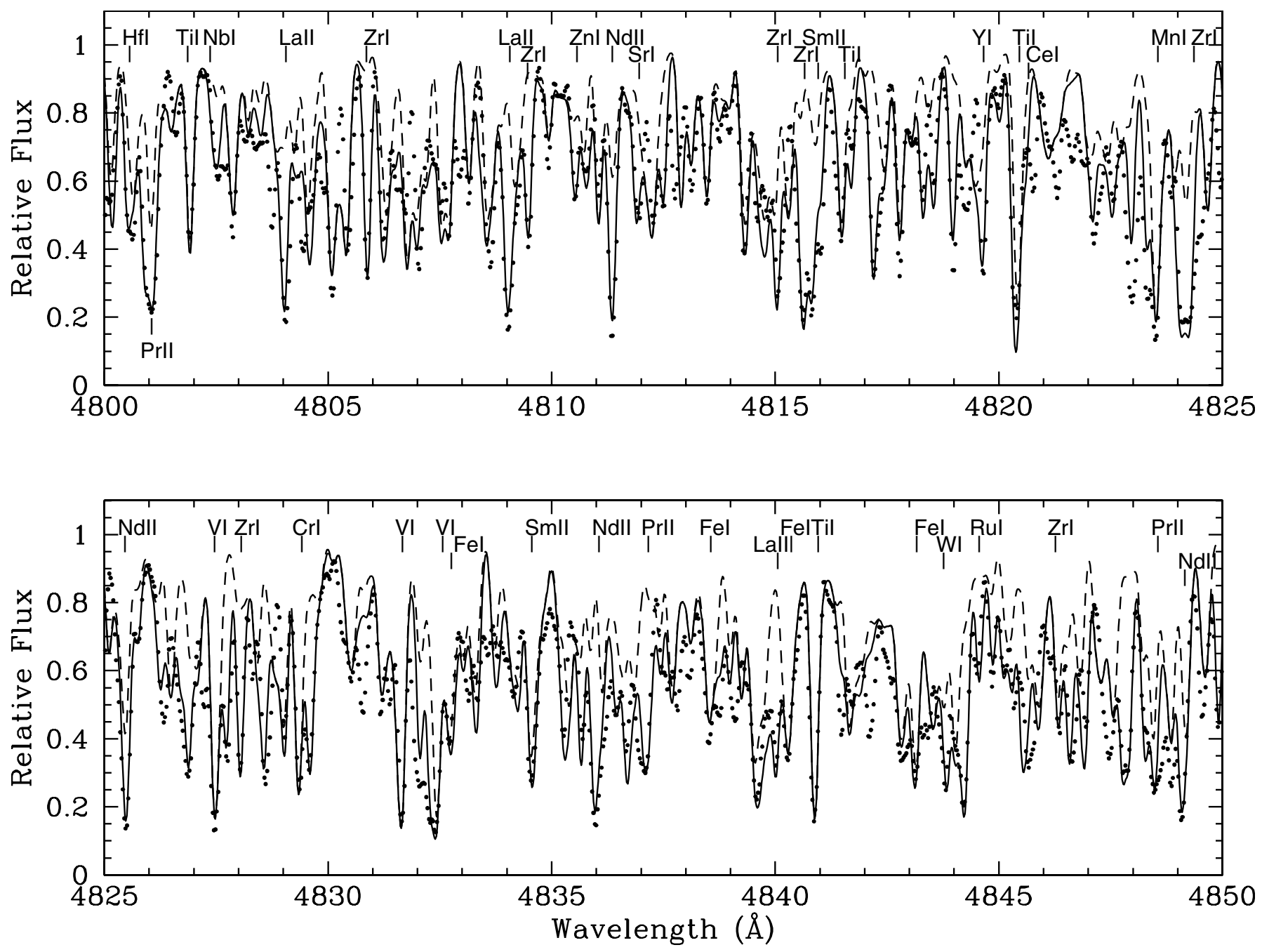

Fig. 2. Synthetic fits to the spectrum of the star IGI95-C1 in the region around $4825 \AA$. From this region we derived most of the s-elements abundances and the mean metallicity of the stars. Some atomic lines, main contributors to the specific spectral feature, are marked. Black dots represent the observed spectrum. Lines are synthetic spectra: only molecules and metals (dashed line), and including s-elements (best fit, continuous line).

studied stars. $\mathrm{Zn}$ abundance is derived from the only accessible line (Zn I at $4810 \AA$ ) which seems to be more sensitive to errors in the atmospheric parameters and damping constant than other $\mathrm{Zn}$ lines (see discussion in Chen et al. 2004). In any case, we estimate a formal error in $[\mathrm{Zn} / \mathrm{M}]$ of 0.25 dex. Keeping in mind this uncertainty, let us recall that AGB stars do not produce a sizable amount of $\mathrm{Zn}$ (Bisterzo et al. 2004; Travaglio et al. 2004), so we can directly compare the abundances measured in our sample of extragalactic carbon stars with that in unevolved galactic stars. For $[\mathrm{Fe} / \mathrm{H}]$ between -1 and -0.5 , unevolved galactic stars show, on the average, $[\mathrm{Zn} / \mathrm{Fe}] \sim+0.15$, with a rather large spread (Mishenina et al. 2002; Bihain et al. 2004; Cayrel et al. 2004; Travaglio et al. 2004). Taking into account this spread and the quoted error bar, we can conclude that the overabundance of $\mathrm{Zn}$ we find in Sagittarius dSph and SMC C-stars does not significantly depart from the figure found for galactic stars of similar metallicity. Note that Reyniers et al. (2004) found $[\mathrm{Zn} / \mathrm{Fe}]=+0.33$ in the galactic post-AGB star IRAS 08143-4406 with $[\mathrm{Fe} / \mathrm{H}]=-0.40$.
The average abundances of the light s-elements (ls: Sr, Y and $\mathrm{Zr}$, corresponding to the first peak of the main component) and that of the heavier s-elements (hs: $\mathrm{Ba}, \mathrm{La}, \mathrm{Nd}$ and Sm, corresponding to the second peak) are reported in Table 5. Ce is not included in the hs definition, because there is no single feature in the spectral ranges studied whose main contributor is $\mathrm{Ce}$ and, our derivation of its abundance is quite uncertain. In addition, we do not include $\mathrm{Sm}$ in the case of IGI95-C3 because it was not measured. The abundance ratios in Table 5 would be not significantly modified if a different continuum location would have been adopted. For instance, we checked if a veil of moderate intensity of some unknown molecular absorption is contributing in this region. It has been found that, considering a continuum placement $\sim 15 \%$ higher, the mean metallicity should be increased by $+0.25 \mathrm{dex}$, the $[\mathrm{s} / \mathrm{M}]$ ratio by +0.30 dex, +0.25 dex for the $[1 \mathrm{~s} / \mathrm{M}]$ ratio, +0.38 dex for the [hs/M] ratio, but only by +0.13 dex in the [hs/ls] ratio. In this case, however, the global fit obtained to the observed spectra in the 4750-4950 $\AA$ region is considerably worse. The same 


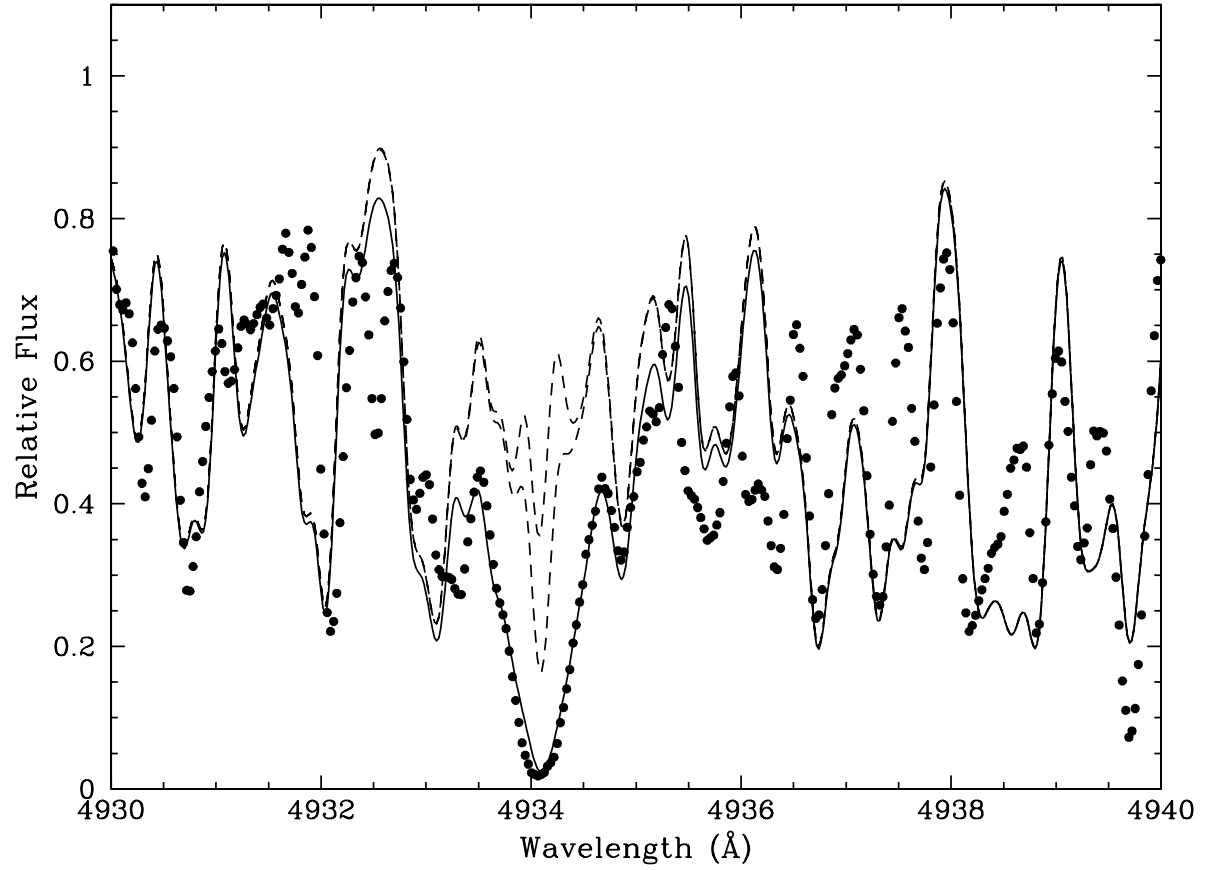

Fig. 3. Synthetic fit to the spectrum of IGI95-C1 in the region of the $\mathrm{Ba}$ II line at $\lambda 4934 \AA$. Black dots are the observed spectrum. Lines are synthetic spectra for different $[\mathrm{Ba} / \mathrm{M}]$ ratios: no $\mathrm{Ba}$ and +0.0 (dashed lines), +1.5 (best fit, continuous line).
Table 5. Metallicity and the s-process indices of the extragalactic C-stars studied. The typical error in $[\mathrm{M} / \mathrm{H}]$ and $[\mathrm{hs} / \mathrm{ls}]$ is $\pm 0.3 \mathrm{dex}$ and the average error on the other indices is \pm 0.35 dex.

\begin{tabular}{cccccc}
\hline \hline Star & {$[\mathrm{M} / \mathrm{H}]$} & {$[\mathrm{s} / \mathrm{M}]$} & {$[\mathrm{ls} / \mathrm{M}]$} & {$[\mathrm{hs} / \mathrm{M}]$} & {$[\mathrm{hs} / \mathrm{ls}]$} \\
\hline IGI95-C1 & -0.8 & 1.1 & 0.6 & 1.5 & 0.9 \\
IGI95-C3 & -0.5 & 1.0 & 1.0 & 1.0 & 0.0 \\
BMB-B 30 & -1.0 & 0.9 & 0.5 & 1.3 & 0.8 \\
\hline
\end{tabular}

test shows that the $\mathrm{C} / \mathrm{O}$ and ${ }^{12} \mathrm{C} /{ }^{13} \mathrm{C}$ ratios would be altered by +0.02 dex and -5 , respectively.

Table 5 shows that the three stars have moderate s-element enhancements, $[\mathrm{s} / \mathrm{M}] \approx+1.0$. This level of enhancement agrees with the figure found in galactic extrinsic (MS, S and $\mathrm{CH}$ types with no Tc) and intrinsic (halo) AGB stars of similar metallicity (see Busso et al. 2001, and references therein). The same happens when comparing the intrinsic index [hs/ls], used to characterize the neutron capture process. Hence, independently of their extrinsic or intrinsic nature (see below), the extragalactic $\mathrm{C}$-stars studied here are similar to their galactic counterparts as far as the s-element enhancements are concerned. On the other hand, comparisons with galactic postAGB stars of low metallicity (Zacs et al. 1995; Reddy et al. 1999; Van Winckel \& Reyniers 2000; Reyniers et al. 2004) show a s-element enhancement typically lower by one order of magnitude.

\section{1. $C$ and $O$ abundances}

Figure 4 shows a theoretical fit of the observed spectrum for the star IGI95-C1 in the spectral region around $8025 \AA$. This spectral region is dominated by $\mathrm{CN}$ absorptions and it has been used to derive the $\mathrm{C} / \mathrm{O}$ and (mainly) the ${ }^{12} \mathrm{C} /{ }^{13} \mathrm{C}$ ratios. The values obtained for our stars are reported in Table 3. Similarly to the figure found in most galactic N-type C-stars (Lambert et al. 1986; Eglitis \& Eglite 1995, 1997; Abia et al. 2002), the derived $\mathrm{C} / \mathrm{O}$ ratios are only slightly larger than 1 . Even considering the uncertainty in the model atmosphere parameters ${ }^{3}$, we do not believe that the spectra of our stars could be reasonably fitted with a $\mathrm{C} / \mathrm{O} \sim 2$, or larger as it is clearly seen in Fig. 4. At solar metallicity, several TP and TDU episodes are required to transform an oxygen-rich $\mathrm{M}$ giant into a carbon star, because of the relatively large $\mathrm{O}$ content in the envelope. When the metallicity is reduced down to $1 / 10$ of the solar value, $\mathrm{C} / \mathrm{O}>1$ is attained after 1 or 2 dredge-up episodes, only. More dredge-up episodes may be required if the original composition was enhanced in oxygen, a possibility that we cannot exclude given the low metallicity of the stars ${ }^{4}$.

It is often claimed that the reason for the recurrence of $\mathrm{C} / \mathrm{O} \sim 1$ in the majority of galactic giant carbon stars may be an observational bias: an excess of carbon in the envelope is immediately translated into a copious production of carbon-rich dust that induces the formation of a thick circumstellar shell obscuring the photosphere at optical wavelengths. In this case, the mass loss rate can reach up to $10^{-5} M_{\odot} / \mathrm{yr}$ and the AGB star rapidly looses its $\mathrm{H}$-rich envelope. Then, visible $\mathrm{C}$-stars represent a short stage of AGB evolution. We also note that even without the star being obscured by dust, the rapid increase in visible and IR gas opacity when C/O passes unity might in itself create a strong levitation which will facilitate the (wind driven) mass loss and quickly bring the stellar life time to

\footnotetext{
${ }^{3}$ As commented in the previous section, the derived $\mathrm{C} / \mathrm{O}$ ratios vary depending on the spectral region analyzed, which probably indicates that something is wrong in the model atmospheres and/or molecular line lists. Note also the possibility of a systematic error due to the uncertain continuum location.

${ }^{4}$ The opposite effect would result if the initial $\mathrm{C}, \mathrm{N}$ and $\mathrm{O}$ abundances in the theoretical models are scaled according to the revised solar values by Asplund et al. (2005).
} 


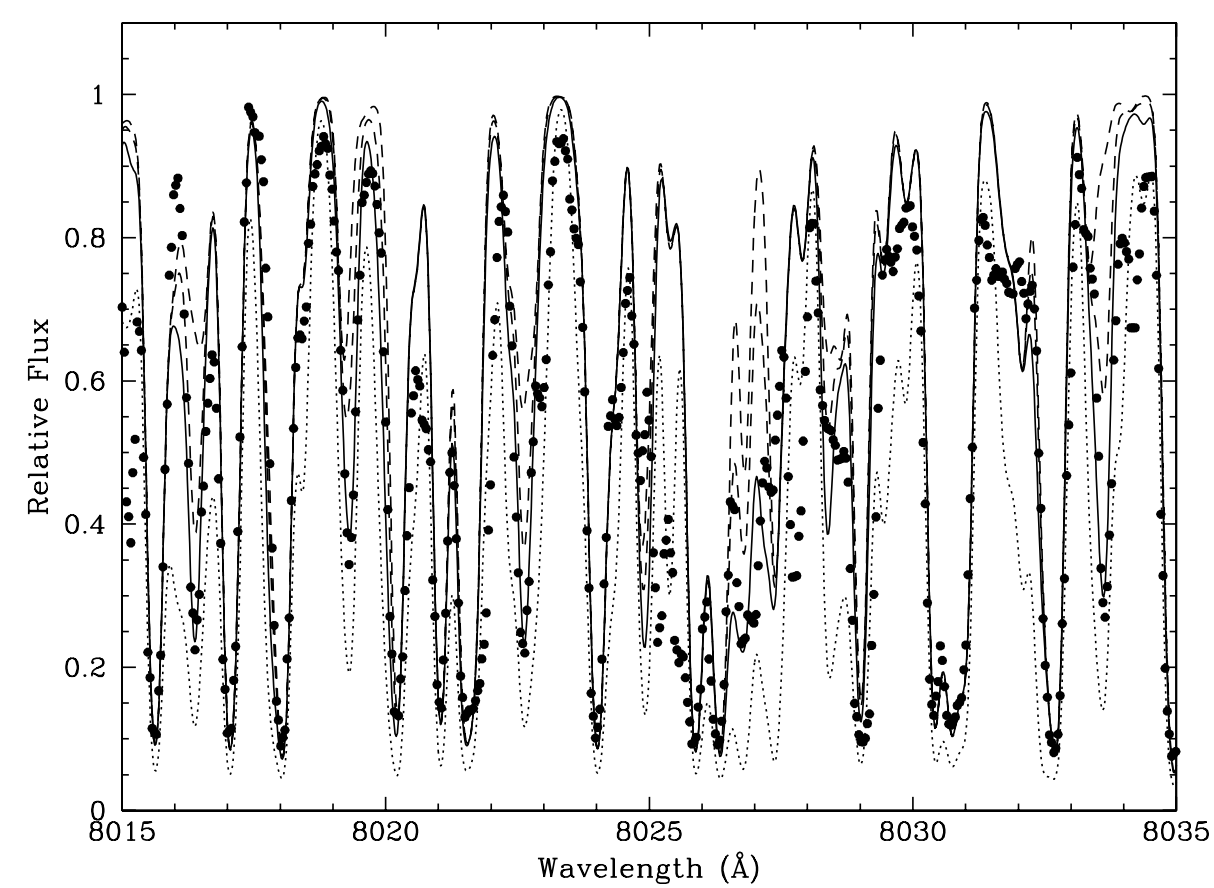

Fig. 4. Synthetic fit to the spectrum of the star IGI95-C1 in the region around $8025 \AA$ from which we derive the ${ }^{12} \mathrm{C} /{ }^{13} \mathrm{C}$ ratio and a first estimate of the $\mathrm{C} / \mathrm{O}$ ratio (see text). The ${ }^{13} \mathrm{C}$ abundance is estimated from the weaker ${ }^{13} \mathrm{CN}$ features at $\lambda \sim 7991,7997$, 8004, 8016, 8019, 8023 and $8034 \AA$. The figure shows only some of these features for clarity. As previous figures, black dots represent the observed spectrum while lines are synthetic spectra for different $\mathrm{C} / \mathrm{O}$ and ${ }^{12} \mathrm{C} /{ }^{13} \mathrm{C}$ ratios. Dashed lines are for no ${ }^{13} \mathrm{C}$ and ${ }^{12} \mathrm{C} /{ }^{13} \mathrm{C}=100$ with $\mathrm{C} / \mathrm{O}=1.18$, respectively; the continuous line is made with the same $\mathrm{C} / \mathrm{O}$ ratio and with our best estimate for the ${ }^{12} \mathrm{C} /{ }^{13} \mathrm{C}$ ratio in this star equal to 25 . The dotted line represents ${ }^{12} \mathrm{C} /{ }^{13} \mathrm{C}=25$ but with $\mathrm{C} / \mathrm{O}=1.80$. Clearly, a $\mathrm{C} / \mathrm{O}$ ratio much larger than unity is discarded. an end. Numerical calculations (Jørgensen \& Johnson 1992) give some support to this theory. However, exceptions seem to exist: Draco 461 is an intrinsic metal-poor C-star belonging to the Draco Spheroidal galaxy which shows $\mathrm{C} / \mathrm{O}=3-5$ (Domínguez et al. 2004). Therefore, if we apply the same reasoning to our sample stars, we should conclude that they have suffered a limited number of TDUs. This might be in contrast with the evidence that also for metal-poor stars, numerous TDU episodes are required to obtain s-element enhancements at the level found in the stars analyzed here (see Table 5). In any case, this figure merits further observational studies in a larger sample of metal-poor C-stars ${ }^{5}$.

Another possible explanation of the low $\mathrm{C} / \mathrm{O}$ may be a partial conversion of the $\mathrm{C}$ dredged up into ${ }^{13} \mathrm{C}$ and ${ }^{14} \mathrm{~N}$ at the bottom of the convective envelope. Intermediate mass AGBs are known to experience a hot bottom burning (e.g. Lattanzio \& Forestini 1999), but in low mass AGBs the temperature at the base of the convective envelope is too low to activate the $\mathrm{CN}$ cycle. Some low mass AGB, however, show evidences of a cool bottom process (Abia et al. 2002; Nollet et al. 2003), i.e. a slow mixing process acting below the convective envelope that may be capable of reducing the $\mathrm{C} / \mathrm{O}$ and the ${ }^{12} \mathrm{C} /{ }^{13} \mathrm{C}$ ratios. An alternative solution for the low $\mathrm{C} / \mathrm{O}$ ratio would arise in the case that our stars were extrinsic C-stars. Abia et al. (2002) showed that, under different assumptions for the dilution factor $^{6}$ the secondary star cannot appear as a carbon $\operatorname{star}(\mathrm{C} / \mathrm{O}>1)$ if the metallicity exceeds $[\mathrm{Fe} / \mathrm{H}] \sim-0.3$. This also implies that extrinsic C-stars with a metallicity slightly lower than this limit

\footnotetext{
${ }^{5}$ On the other hand, it would be interesting to derive the $\mathrm{C} / \mathrm{O}$ ratios in a large sample of $\mathrm{CH}$ stars; the only metal-poor (extrinsic) carbon stars where the $\mathrm{C} / \mathrm{O}$ ratios found are considerably larger than unity (Vanture 1992).

${ }^{6}$ The dilution factor is defined as the ratio of the mass transferred from the primary star to the envelope mass of the accreting secondary star, supposed to have the original or post first dredge-up composition.
}

should have $\mathrm{C} / \mathrm{O}$ ratios not too much larger than unity, in agreement with our finding. This is not a proof of the extrinsic nature of our sample stars, but simply indicates that this hypothesis is compatible with the measured $\mathrm{C} / \mathrm{O}$ ratios. We will come back to this point later, but note that according to the estimations by Abia et al. (2002) (see their Table 6), this hypothesis requires that the $\mathrm{C} / \mathrm{O}$ ratio in the primary star at the mass-transfer epoch should have been larger than about 5 (the exact value depends on the metallicity). As far as we know, only a few planetary nebulae has already been observed with such a large $\mathrm{C} / \mathrm{O}$ ratio.

A second remarkable result, which is related to the $\mathrm{C} / \mathrm{O}$ ratio, concerns the ${ }^{12} \mathrm{C} /{ }^{13} \mathrm{C}$ ratio. In two of the stars studied here we find relatively low carbon isotopic ratios $(\approx 20-40)$, similar to those found in many galactic carbon stars of nearly solar metallicity (see e.g. Abia et al. 2003, and references therein). The observed ${ }^{12} \mathrm{C} /{ }^{13} \mathrm{C}$ ratio in galactic RGB stars of low metallicity is near 7 (Gilroy \& Brown 1991; Gratton et al. 2000). As a consequence of the TDU, the carbon isotopic ratio is expected to increase during the TP-AGB phase. Then, the values measured in the case of IGI95-C1 and IGI95-C3, namely about 5 times larger than the typical value found in bright red giant stars, are compatible with the ${ }^{12} \mathrm{C}$ enhancement implied by their $\mathrm{C} / \mathrm{O} \approx 1$. This is also valid in the case of mass transfer and dilution (extrinsic C-stars). In contrast to this, the very high carbon isotopic ratio found in BMB-B 30 (>300) would require a much higher $\mathrm{C} / \mathrm{O}$ ratio than the one we derive. There are no obvious solutions for this problem, namely how to keep the $\mathrm{C} / \mathrm{O}$ ratio close to unity without decreasing the ${ }^{12} \mathrm{C} /{ }^{13} \mathrm{C}$ ratio. A possibility would be the existence of a deep enough convective intershell region during the thermal pulse. This would mix $\mathrm{O}$ (in addition to $\mathrm{C}$ ) up to the top of the intershell. Then, the subsequent normal TDU may bring $\mathrm{C}$ and $\mathrm{O}$ to the surface, reducing the increase of the $\mathrm{C} / \mathrm{O}$ ratio. However, there are at least two important consequences of this. First, because the temperature at the base of the convective TP would be as large as 


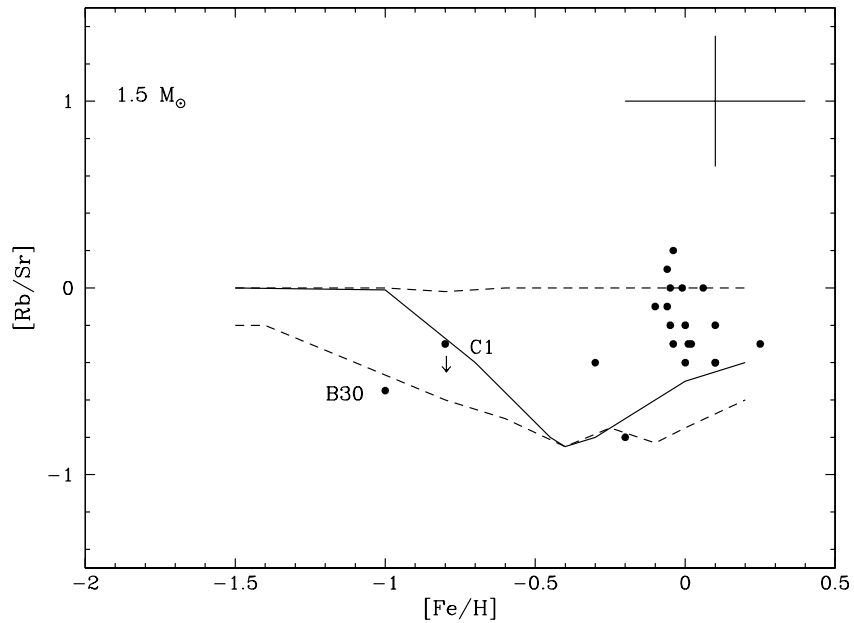

Fig. 5. Comparison of the observed $[\mathrm{Rb} / \mathrm{Sr}]$ ratio vs. $[\mathrm{M} / \mathrm{H}]$ with the theoretical predictions for different choices of the ${ }^{13} \mathrm{C}$-pocket (see text) for a $1.5 M_{\odot}$ TP-AGB. Continuous line represents the ST (standard) choice while dashed lines limit the area allowed by the choices from ST $\times 2$ to ST/12. Theoretical models are from Gallino et al. (1998). The stars studied here are marked. The abundance ratios corresponding to IGI95-C3 are not shown because we do not detected $\mathrm{Rb}$ in this star. Similar ratios derived in galactic N-type carbon stars (Abia et al. 2001) (black dots at near solar metallicity) are also shown for comparison.

$3.5 \times 10^{8} \mathrm{~K}$, then one would expect an s-element pattern altered according to the activation of the ${ }^{22} \mathrm{Ne}$ neutron source. Second, since the TDU of carbon would be very strong, the ${ }^{12} \mathrm{C} /{ }^{13} \mathrm{C}$ ratio should be very large ( 500-1000). Leaving apart that this possibility may have also important consequences for the subsequent evolution of the star, the s-element pattern found in this star (see Tables 4, 5 and Fig. 5) is at odds with this possibility.

From the previous discussion, it is seen that the $\mathrm{C}-\mathrm{N}-\mathrm{O}$ abundances are key to understanding AGB evolution and nucleosynthesis. Additional observational studies in a large sample of stars are required, preferably with different indicators, such as the CNO-bearing molecular bands in the infrared, which seem to be less prone to line formation problems like saturation or blends.

\subsection{Heavy elements}

The relative abundances of s-elements that follow the ${ }^{85} \mathrm{Kr}$ reaction branching ( $\mathrm{Rb}, \mathrm{Sr}, \mathrm{Zr}$ and $\mathrm{Y}$ ) along the s-process path yield information on the neutron density prevailing during the neutron capture processes occurring in the He intershell (Beer \& Macklin 1989; Lambert et al. 1995; Abia et al. 2001). In particular, the $[\mathrm{Rb} / \mathrm{Sr}]$ helps to discriminate between the two main neutron sources, the ${ }^{13} \mathrm{C}(\alpha, n){ }^{16} \mathrm{O}$ and ${ }^{22} \mathrm{Ne}(\alpha, n){ }^{25} \mathrm{Mg}$, operating in TP-AGB stars. The former dominates the s-process nucleosynthesis in low mass AGBs $\left(M<3 M_{\odot}\right)$ for which $[\mathrm{Rb} / \mathrm{Sr}]<0$ is expected. Figure 5 shows the comparison of the $[\mathrm{Rb} / \mathrm{Sr}]$ ratios derived in BMB-B 30 and IGI95-C1 (no detection for IGI95-C3) with s-process model predictions for a 1.5 $M_{\odot} \mathrm{AGB}$ star at $\mathrm{C} / \mathrm{O}>1$ (Gallino et al. 1998; Busso et al. 1999, 2004). The same ratios derived in galactic N-type C-stars are also shown for comparison. The lines in Fig. 5

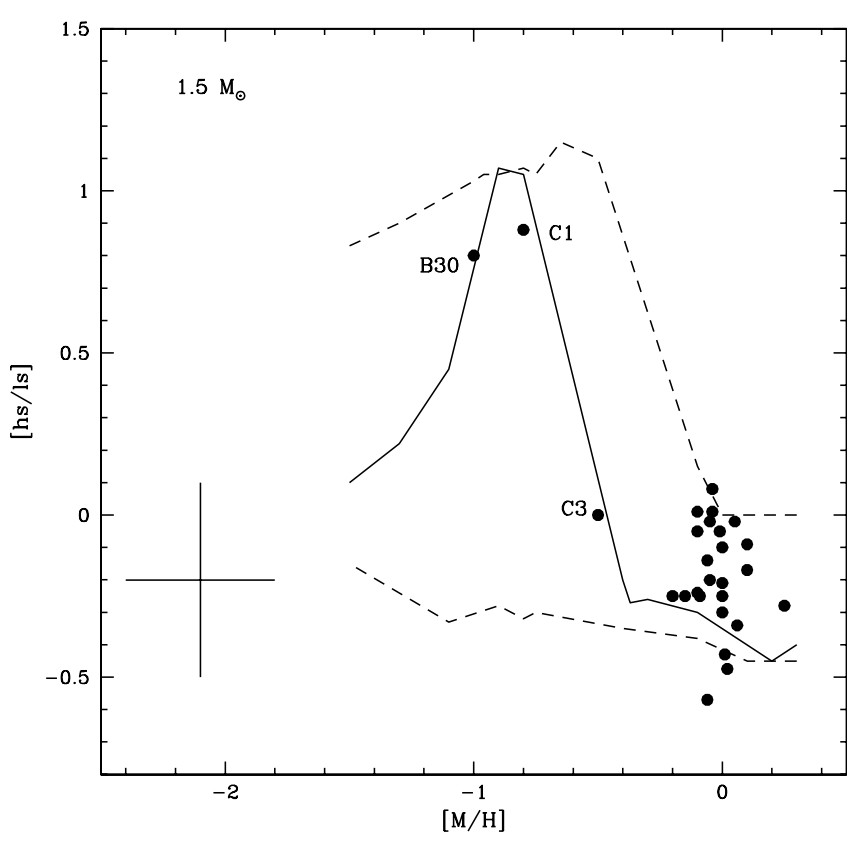

Fig. 6. Comparison of the observed mean of heavy (Ba, La, Nd and $\mathrm{Sm}$ ) to light-mass ( $\mathrm{Sr}, \mathrm{Y}$ and $\mathrm{Zr}$ ) s-element [hs/ls] enhancement (signature of the neutron exposure) against metallicity with theoretical prediction for a $1.5 M_{\odot}$ TP-AGB. Continuous and dashed lines represent the same as Fig. 5. The location of the stars studied here are marked. At near solar metallicity, the galactic carbon stars studied in Abia et al. (2002, after minor revisions) are shown for comparison.

correspond to a specific assumption about the ${ }^{13} \mathrm{C}$ abundance in the ${ }^{13} \mathrm{C}$-pocket. We show the case defined as standard (ST) by Gallino et al. (1998), corresponding to $4 \times 10^{-6} M_{\odot}$ of ${ }^{13} \mathrm{C}^{7}$. The derived ratios are in good agreement with the model predictions and are similar to those obtained for galactic N-type Cstars. Comparison of the derived $[\mathrm{Rb} / \mathrm{Zr}, \mathrm{Y}]$ ratios with model predictions leads to the same conclusion.

Figure 6 reports the intrinsic index [hs/ls] ratio in our stars in comparison with model predictions for a $1.5 M_{\odot}$ AGB star at the beginning of the TP-AGB phase (the same models as Fig. 5). The solid line represents the standard choice for the amount of ${ }^{13} \mathrm{C}$ in the ${ }^{13} \mathrm{C}$-pocket (ST case). Similar abundance ratios found in $\mathrm{N}$-type galactic $\mathrm{C}$-stars (Abia et al. 2002) are also plotted. At high (nearly solar) metallicity the light-s (ls) are slightly overproduced with respect to the Ba group elements (hs). The [hs/ls] ratio increases with decreasing metallicity as more neutrons are available per seed. A maximum is reached around $[\mathrm{Fe} / \mathrm{H}] \sim-1$, a metallicity within the range spanned by our sample of stars. Then, at very low metallicity $([\mathrm{Fe} / \mathrm{H}] \leq-2)$ and at the end of the TP-AGB phase, the [hs/ls] tends toward a constant value, since most of the neutrons are spent to produce lead (see e.g. Busso et al. 1999). The three studied stars seem to follow the variation with the metallicity of the standard choice of the ${ }^{13} \mathrm{C}$-pocket (ST case). Obviously, the large uncertainty in the abundance ratios and the scarcity of data at low metallicity prevent us from concluding whether

${ }^{7}$ For a more detailed discussion of the models see Gallino et al. (1998). 
there is a preferred average abundance of ${ }^{13} \mathrm{C}$ (or spread) with a different effect at different metallicities.

To return to the question of the extrinsic or intrinsic nature of our stars, as it is well known, the detection of Tc is an undeniable evidence that a star, with s-element enhanced composition, is currently undergoing TDU. The only Tc isotope with a sufficiently long life to be observable is indeed ${ }^{99} \mathrm{Tc}$, whose decay lifetime ${ }^{8}$ is comparable to the time elapsed between two subsequent TPs in low mass AGB stars. This Tc isotope is produced by the s-process and it is expected to appear at the surface of a TP-AGB star undergoing TDU. Unfortunately, our instrument set-up did not include the adequate spectral regions for Tc detection. However, an alternative test of the intrinsic nature of a C-star is provided by the measurement of $\mathrm{Nb}$ abundance, a chemical element with only one stable isotope,${ }^{93} \mathrm{Nb}$, which can be produced by ${ }^{93} \mathrm{Zr}$ decay ${ }^{9}$. The ${ }^{93} \mathrm{Zr}$ lifetime is comparable with the duration of the whole AGB phase and, thus, the comparison of the abundance of $\mathrm{Nb}$ and that of its neighbors (e.g. Zr, Y) would tell us if the s-process enhancement is intrinsic or due to mass transfer. S-process nucleosynthesis calculations predict that the $\mathrm{Zr}$ isotopes and, in particular ${ }^{93} \mathrm{Zr}$, increase during the AGB phase, while $\mathrm{Nb}$ remains almost unchanged (Straniero et al. 2005). As a consequence, when the star attains the C-rich star stage, $[\mathrm{Nb} / \mathrm{Zr}] \sim-0.5$, almost independently of its metallicity. After a few million years, however, as a consequence of the ${ }^{93} \mathrm{Zr}$ decay, this ratio rises to the scaled solar value. In other words, for an extrinsic C-star $[\mathrm{Nb} / \mathrm{Zr}]$ should be $\sim 0$. From our spectra we were only able to derive upper limits, $[\mathrm{Nb} / \mathrm{Zr}]<-0.4 \pm 0.4$, for all the three studied C-stars. This figure would hence indicate that they are probably intrinsic C-stars. A better determination of the $\mathrm{Nb}$ abundance is however needed to give a definitive answer.

\section{Conclusions}

In this paper we have presented for the first time a detailed chemical analysis of three extragalactic carbon stars, focusing mainly on their s-element content and its dependence on metallicity. The three stars are moderately metal-poor with a mean metallicity compatible with the typical metallicity observed in the main stellar population of the respective host satellite galaxy, namely: the SMC and Sgr dSph. All the stars show an s-element enhancement of $[\mathrm{s} / \mathrm{M}] \approx+1.0$, which is of the same order as that found in galactic AGB stars of similar metallicity. The s-element abundance pattern found across the ${ }^{85} \mathrm{Kr}$ branching point and the second s-peak (Ba group elements) of the s-path can be understood on the basis of theoretical nucleosynthesis models, if the stars have low masses $\left(M<3.0 M_{\odot}\right)$, and the ${ }^{13} \mathrm{C}(\alpha, n){ }^{16} \mathrm{O}$ reaction is the main source of neutrons. The derived intrinsic index $[\mathrm{hs} / \mathrm{ls}]$ in the three stars seems to be in agreement with a preferred choice for the abundance of ${ }^{13} \mathrm{C}$ that is burnt in stars with different metallicity. However, given the large error bars in the abundances and the limited number of stars analysed here, our results are not in contradiction with the figure extracted from studies in galactic AGB and post-AGB

\footnotetext{
8 The value is $\tau_{1 / 2} \sim 2 \times 10^{5} \mathrm{yr}$.

${ }^{9}$ Half-life $\tau_{1 / 2} \sim 1.5 \times 10^{6}$ yr.
}

stars concerning the existence of a dispersion in the amount of ${ }^{13} \mathrm{C}$ burnt in stars with different metallicity. Obviously, more extended studies with narrower error bars on the derived abundances are needed to confirm this. As for galactic C-stars, the $\mathrm{C} / \mathrm{O}$ ratios derived in these three extragalactic $\mathrm{C}$-stars are found to be only slightly larger than unity. This fact, together with the derived ${ }^{12} \mathrm{C} /{ }^{13} \mathrm{C}$ ratios and the large s-process enhancement, is in disagreement with theoretical predictions for TP-AGB stars.

Acknowledgements. We warmly thank B. Gustafsson for his careful reading of the manuscript and his valuable suggestions that considerably improved this work. The referee, H. Van Winckel, is thanked for his useful comments. This publication makes use of data products from the Two Micron All Sky Survey, which is a joint project of the University of Massachusetts and the Infrared Processing and Analysis Center/California Institute of Technology, funded by the National Aeronautics and Space Administration and the National Science Foundation. Part of this work was supported by the Spanish grant AYA2002-04094-C03-03 from the MCyT.

\section{References}

Aaronson, M., \& Mould, J. 1985, ApJ, 290, 191

Abia, C., Boffin, H. M. J., Isern, J., et al. 1993, A\&A, 272, 455

Abia, C., \& Isern, J. 1996, ApJ, 460, 443

Abia, C., Busso, M., Gallino, R., et al. 2001, ApJ, 559, 1117

Abia, C., Domínguez, I., Gallino, R., et al. 2002, ApJ, 578, 817

Abia, C., Domínguez, I., Gallino, R., et al. 2003, PASA, 20, 314

Alknis, A., Balnauss, A., Dzervitis, V., \& Eglitis, I. 1998, A\&A, 338, 209

Alvarez, R., \& Plez, B. 1998, A\&A, 330, 1109

Asplund, M., Grevesse, N., Sauval, A. J., et al. 2005, A\&A, 431, 693

Aoki, W., Ryan, S. G., Norris, J. E., et al. 2002, ApJ, 580, 1149

Arimoto, N., Ikuta, C., \& Jablonka, P. 2004, in Studies of Galaxies in the Young Universe with New Generation of Telescope, ed. N. Arimoto, \& W. Duschl, 147

Bihain, G., Israelian, G., Rebolo, R., Bonifacio, P., \& Molaro, P. 2004, A\&A, 423, 777

Blanco, V. M., Blanco, B. M., \& McCarthy, M. F. 1980, ApJ, 242, 938

Battinelli, P., Demers, S., \& Letarte, B. 2003, A\&A, 405, 563

Battinelli, P., \& Demers, S. 2004, A\&A, 418, 33

Barnbaum, C., Stone, R. P. S., \& Keenan, P. C. 1996, ApSS, 105, 419

Beer, H., \& Macklin, R. L. 1989, ApJ, 339, 962

Bergeat, J., Knapik, A., \& Rutily, B. 2001, A\&A, 369, 178

Bisterzo, S., Gallino, R., Pignatari, M., et al. 2004, MmSAI, 75, 741

Bonifacio, P., et al. 2004b, in ESO Workshop in Chemical Evolution and Mixing in Stars in the Milky Way and its Satellites, ed. S. Randich, in press

Bonifacio, P., Sbordone, L., Marconi, G., et al. 2004a, A\&A, 414, 503

Bullock, J. S., Kravtsov, A. V., \& Weimberg, D. H. 2001, ApJ, 548, 33

Busso, M., Gallino, R., \& Wasserburg, G. 1999, ARA\&A, 37, 329

Busso, M., Lambert, D. L., Gallino, R., Travaglio, C., \& Smith, V. V. 2001, ApJ, 557, 802

Busso, M., Straniero, O., Gallino, R., \& Abia, C. 2004, in Origin and Evolution of the Elements, ed. A. McWilliam, \& M. Rauch (Cambridge University Press), 68

Cayrel, R., Depagne, E., Spite, M., et al. 2004, A\&A, 416, 1117

Chen, Y. Q., Nissen, P. E., \& Zhao, G. 2004, A\&A, 425, 697

Cristallo, S., et al. 2005, in preparation

Cioni, M.-R. L., van der Marel, R. P., Loup, C., et al. 2000, A\&A, 359, 601

Decin, L., Waelkens, C., Eriksson, K., et al. 2000, A\&A, 364, 137 
Delaude, D., Gallino, R., Cristallo, S., et al. 2004, MmSAI, 75, 706

Den Hartog, E. A., Lawler, J. E., Sneden, C., \& Cowan, J. J. 2003, ApJS, 148, 543

Demers, S., Battinelli, P., \& Latarte, B. 2003, AJ, 125, 3037

Denn, G. R., Luck, R. E., \& Lambert, D. L. 1991, ApJ, 377, 657

Domínguez, I., Abia, C., Straniero, O., Cristallo, S., \& Pavlenko, Y. 2004, A\&A, 422, 1045

Eglitis, I., \& Eglite, M. 1995, Ap\&SS, 229, 63

Eglitis, I., \& Eglite, M. 1997, Ap\&SS, 249, 235

Gallino, R., Arlandini, C., Busso, M., et al. 1998, ApJ, 497, 388

Goriely, S., \& Siess, L. 2001, A\&A, 378, L25

Gratton, R., Sneden, C., Carretta, E., \& Bragaglia, A. 2000, A\&A, 354, 169

Gilroy, K., \& Brown, J. A. 1991, ApJ, 371, 578

Goriely, S., \& Siess, L. 2001, A\&A, 378, L25

Grebel, E. K., Gallagher, J. S., \& Harbeck, D. 2003, ApJ, 125, 1926

Grevesse, N., \& Sauval, A. J. 1998, Space Sci. Rev., 85, 161

Groenewegen, M. A. T. 2002, in The Chemical Evolution of Dwarf Galaxies [arXiv:astro-ph/0208449]

Groenewegen, M. A. T. 2004 [arXiv:astro-ph/047282]

Guandalini, R., Busso, M., Ciprini, S., et al. 2006, A\&A, 445, 1069

Gustafsson, B., et al. 2005, in preparation

Herwig, F., Blöcker, T., Schönberner, D., \& El Eid, M. 1997, A\&A, 324, L81

Hill, V., Plez, B., Cayrel, R., et al. 2002, A\&A, 387, 560

Holweger, H., \& Müller, E. A. 1974, Sol. Phys., 39, 19

Ibata, R. A., Gilmore, G., \& Irwin, M. J. 1995, MNRAS, 277, 781

Iben, I. Jr., \& Renzini, A. 1983, ARA\&A, 21, 271

Jørgensen, U. G., \& Johnson, H. R. 1992, A\&A, 265, 168

Jørgensen, U. G., et al. 2005, in preparation

Kupka, F., Piskunov, N. E., Ryabchikova, T. A., Stempels, H. C., \& Weiss, W. W. 1999, A\&AS, 138, 119

Lambert, D. L., Gustafsson, B., Eriksson, K., \& Hinkle, K. H. 1986, ApJS, 62, 373

Lambert, D. L., Smith, V. V., Busso, M., Gallino, R., \& Straniero, O. 1995, ApJ, 450, 302

Lattanzio, J., \& Forestini, M. 1999, in Asymptotic Giant Branch Stars, ed. T. Le Bertre, A. Lèbre, \& C. Waelkens, IAU Symp., 191, 31

Luck, R. E., Moffet, R. J., Barnes, T. G., et al. 1998, AJ, 115, 605

McKellar, A. 1947, JRASC, 41, 147

Mishenina, T. V., Koutyukh, V. V., Soubiran, C., Travaglio, C., \& Busso, M. 2002, A\&A, 396, 189

Monaco, L., Bellazzini, M., Ferraro, F. R., et al. 2004, MNRAS, 353 , 874

Morgan, D. H., Hatzidimitriou, D., \& Cannon, R. D. 2004, MNRAS, in press
Nollet, K. N., Busso, M., \& Wasserburg, G. 2003, ApJ, 582, 1036

Paczynski, B. 1970, Acta Astronomica, 20, 47

Peterson, R. C., Dalle Ore, C. M., \& Kurucz, R. L. 1993, ApJ, 404, 333

Plez, B., \& Cohen, J. G. 2005, A\&A, 434, 1117

Querci, F., Querci, M., \& Kunde, V. G. 1971, A\&A, 15, 256

Rebeirot, E., Azzopardi, M., \& Westerlund, B. E. 1993, A\&AS, 97, 603

Reddy, B. E., Bakker, E. J., \& Hrivnak, B. J. 1999, ApJ, 524, 831

Reyniers, M., Van Winckel, H., Gallino, R., \& Straniero, O. 2004 A\&A, 417, 269

Rizzi, L., Held, E. V., Bertelli, G., \& Saviane, L. 2004, MmSAI, 75, 110

Rolleston, W. R. J., Dufton, P. L., McErlean, N. D., et al. 1999, A\&A, 348,728

Sarre, P. J., Hurst, M. E., \& Lloyd Evans, T. 2000, MNRAS, 319, 103

Schlegel, D. J., Finkbeiner, D. P., \& Davis, M. 1998, ApJ, 500, 525

Shetrone, M. D., Bolte, M., \& Stetson, P. B. 1998, ApJ, 115, 1888

Smith, V. V., Plez, B., Lambert, D. L., \& Lubowich, D. A. 1995, ApJ, 441,735

Straniero, O., Gallino, R., Busso, M., et al. 1995, ApJ, 440, L85

Straniero, O., Chieffi, A., Limongi, M., et al. 1997, ApJ, 478, 332

Straniero, O., Domínguez, I., Cristallo, S., \& Gallino, R. 2003, PASA, 20,389

Straniero, O., Gallino, R., \& Cristallo, S. 2005, Nucl. Phys. A, in press

Thévenin, F. 1989, A\&AS, 77, 137

Thévenin, F. 1990, A\&AS, 82, 179

Travaglio, C., Gallino, R., Arnone, E., et al. 2004, ApJ, 601, 864

Van Eck, S., Goriely, S., Jorissen, A., \& Plez, B. 2001, Nature, 412, 793

Van Eck, S., Goriely, S., Jorissen, A., \& Plez, B. 2003, A\&A, 404, 291

Van Winckel, H., \& Reyniers, M. 2000, A\&A, 354, 135

Vanture, A. J. 1992, AJ, 103, 2035

Venn, K., Irwin, M., Shetrone, M. D., et al. 2004, AJ, 128, 1177

Whitelock, P. A., Irwin, M., \& Catchpole, R. M. 1996, New Astronomy, 1, 57

Wallerstein, G., \& Knapp, G. R. 1998, ARA\&A, 36, 369

Wasserburg, G. J., Boothroyd, A. I., \& Sackmann, I.-J. 1995, ApJ, 440, L101

Wyller, A. A. 1966, ApJ, 143, 828

Wood, P. R., Bessell, M. S., \& Fox, M. W. 1983, ApJ, 272, 99

Xu, H. L., Svanberg, S., Cowan, R. D., et al. 2003, MNRAS, 346, 433

Yamashita, Y., \& Utsumi, K. 1967, PASJ, 20, 73

Zacs, L., Klochkova, V. G., \& Panchuk, V. E. 1995, MNRAS, 275, 764

Zinner, E. 1998, Annu. Rev. Earth. Planet. Sci., 26, 147 